\title{
Numerical characterization of the density of metastable states within the hysteresis loop in disordered systems
}

Joël Puibasset

Centre de Recherche sur la Matière Divisée, CNRS-Université d'Orléans, $1 \mathrm{~b}$ rue de la Férollerie, 45071 Orléans cedex 02, France

E-mail : puibasset@cnrs-orleans.fr

\begin{abstract}
An improved approach is proposed to analyze the density of metastable states within any hysteresis loop, as those observed in magnetic materials or for adsorption in porous materials. Except for few analytically tractable models, most calculations have to be performed numerically on finite systems. The main points to be addressed thus concern the average over various material samples (the socalled realizations of the disorder), and the finite size analysis to estimate the thermodynamic limit. As an improvement of previously existing methods, it is proposed to introduce the Fourier transform of the density of metastable states (characteristic function). Its logarithm is shown to be additive and can straightforwardly be averaged over disorder. This procedure leads to a new definition of the complexity in finite size, giving the usual quenched complexity in the thermodynamic limit, while being better suited to perform finite size analysis.
\end{abstract}


The calculations are illustrated on a molecular simulation based model for a simple fluid adsorbed in heterogeneous siliceous tubular pores mimicking mesoporous materials like MCM-41 or porous silicon. It is expected to be of general interest for hysteresis phenomena, including magnetic materials. 


\section{Introduction}

Hysteresis appears in many situations, the most frequent being magnetism. It is also found for fluids confined in nanopores: for some experimental conditions, the fluid adsorption and desorption isotherms may differ, and exhibit a hysteresis. Puzzling experimental observations have not yet been accounted for by a molecular approach. For instance, one observes large hysteresis in regular mesopores even when they are closed at one end. ${ }^{1-3}$ Wallacher and collaborators $^{4}$ suggested that the hysteresis emerges as a consequence of intrinsic disorder within the pores. The implication of such a disorder to explain hysteresis was shown in structures like aerogels. ${ }^{5-7}$ Taking into account the effect of physico-chemical heterogeneities on fluid adsorption, ${ }^{8}$ and the interconnections between the elementary domains delimited by these heterogeneities, we arrived at the conclusion that these heterogeneities within pores give rise to a large hysteresis loop with a triangular shape. ${ }^{9,10}$ A simplified lattice gas approach gives similar conclusions. ${ }^{11}$

The boundary of the hysteresis corresponds to the main adsorption and desorption branches. As for magnetism, the inner region of the loop can be explored by performing incomplete adsorption/desorption cycles (scanning curves). This shows the existence of a plethora of metastable states and gives some information regarding their organization. The same procedure could be used in our numerical experiment. However, it misses many metastable states, as can be shown by changing the size of the reservoir. ${ }^{12}$ We thus perform a systematic exploration of all metastable states within the hysteresis. These metastable states arise as a consequence of the complex free energy landscape which is ubiquitously observed in systems exhibiting disorder. In our case, this disorder is a consequence of the strong heterogeneity in the physico-chemical properties of the inner surface of the porous material. 
An interesting issue is to characterize the density of metastable states within the hysteresis loop. Such studies have a long history in magnetic systems, but recent works have shown their relevance to adsorption hysteresis. ${ }^{13,14}$ Previous works in both fields have shown that this density grows exponentially with system size inside the loop. One thus introduces the complexity, i.e. the logarithm of the density normalized to the system size. This quantity depends on the spatial distribution of the heterogeneities in the pore, and one has to somehow perform an average over all possible pore realizations. This is however not immediate since the average may be performed either on the density of metastable states, leading to the socalled annealed complexity, or on the complexity itself, leading to the so-called quenched complexity. The annealed complexity, which characterizes the average density of metastable states, is unsuited to describe the hysteresis loop in the thermodynamic limit. ${ }^{13}$ On the other hand, the quenched complexity, which characterizes the typical density of metastable states, is expected to lead to the correct thermodynamic limit, and has been extensively used to characterize the adsorption/desorption hysteresis loop. ${ }^{13-15}$

Another approach may however be proposed, based on the characteristic function associated to the density of metastable states. We show that its logarithm is straightforwardly averaged over disorder, providing a natural characterization of the distribution of metastable states in terms of average over disorder. This approach is shown to be in close connection with the previous one, leading to the usual quenched complexity in the thermodynamic limit. The calculations are more involved and more sensitive to numerical inaccuracies, which may explain why it has never been introduced in this context. This method is however shown to exhibit a remarkably faster convergence in terms of finite size analysis, because it relies on an exact description of the growth of the density of metastable states with system size when 
subsystems become essentially independent. From this point of view, it proves to be particularly suited if one is interested in numerical extrapolations to the thermodynamic limit. These results have been obtained in the context of fluid adsorption in inhomogeneous linear pores to be described hereafter, but are expected to be quite general.

The paper is organized as follows: After a presentation of the molecular model and the standard methods, we introduce the approach exploiting the properties of the characteristic function associated to the distribution of metastable states. We finally show that this method is able to provide quantitative information regarding the quenched complexity in the thermodynamic limit. The last section summarizes and gives some conclusions.

\section{Model and methods}

Our model is designed to catch the main features of simple fluid adsorption in heterogeneous siliceous linear nanopores ( $3 \mathrm{~nm}$ in diameter). It can be seen as a simplified model for MCM41 or oxidized porous silicon, if one admits that these materials exhibit corrugated pores. This is however a highly debated issue and alternative models have been proposed. ${ }^{16-18}$ The results are however expected to be transferable to any other system exhibiting heterogeneous nanopores, like for instance the nanoporous alumina, ${ }^{2,3}$ or pores modulated at mesoscopic scale. ${ }^{19}$ All interactions are supposed to follow the Lennard-Jones $(12,6)$ potential. The parameters are those for argon-argon and argon-oxygen species to describe fluid-fluid and fluid-silica interactions. ${ }^{9}$ Heterogeneities arise as a modulation of the fluid/wall interaction along the pore axis: this results in a string of more or less attractive domains (see Fig. 1). In our model, we chose domains to be $7 \mathrm{~nm}$ long. Due to short range interactions (less than the 
domain length) and rapid decrease of correlations, the fluid properties in a given domain are essentially determined by the imposed chemical potential $\mu$ and the fluid state (density) in the two neighboring domains (the first and last domains are in contact with the bulk gas state since pores are supposed to be open at both ends). For a given $\mu$, the number of such fluid states (densities) is actually quite small, which allows considering all of them to calculate all "conditional" isotherms of the domain under consideration. The fluid confined in a domain may either be in the gaslike state, denoted G, corresponding to the fluid adsorbed only at the walls, or in the liquidlike state, denoted L, corresponding to the fluid filling the whole domain. This gives at most four conditional isotherms for each domain. After these calculations are performed for all domains, the fluid properties can be determined in the whole pore for any chemical potential path. This approach thus allows a decoupling between the atomistic level calculations performed by molecular simulation in each domain (Grand Canonical Monte Carlo), and the large-scale calculation of the fluid state in the heterogeneous pore of micrometric length. It is emphasized that this is not a simple independent domain approach since the strong interaction between adjacent domains are taken into account. More details on the model can be found in refs $\left[{ }^{9}\right]$ and $\left[{ }^{10}\right]$. For instance the main adsorption isotherm, from vacuum to saturation, is calculated as follows: at low $\mu$, all domains are in the gaslike state and follow the gaslike branch of its G-G conditional isotherm until one domain reaches its limit of stability and jumps to the liquidlike state. This obviously impacts the fluid state in the neighboring domains with a possible avalanche, until a new self-consistent state is reached. This avalanche corresponds to the propagation of a liquid meniscus. The chemical potential is further increased, and the same procedure is applied until saturation is reached. Desorption and scanning isotherms are obtained in a similar way. 
As explained in the Introduction, we need to determine all the possible metastable states for the confined fluid. Let us consider a pore with $N$ domains. At chemical potential $\mu$, the fluid may be in one of two states ( $\mathrm{G}$ or $\mathrm{L}$ ) for each domain. One then expects at most $2^{N}$ possible fluid states, which allows a systematic analysis for small enough $N$. Most of these configurations are actually impossible due to the interdependence between neighboring domains. Our algorithm selects the few ones that are consistent with the conditional isotherms. These configurations are stable against thermal fluctuations (taken into account in the pre-calculated isotherms) and correspond to the metastable states (see Fig. 2). It was shown in previous work that the number of metastable states grows exponentially with system size but remains much smaller than $2^{N}$. The density $\mathcal{N}(\rho ; \mu ; N)$ of metastable states in the $\mu$ $\rho$ plane is defined so that $\mathcal{N}(\rho ; \mu ; N) d \rho$ is, for a given chemical potential $\mu$, the number of metastable states in the interval $d \rho$ around $\rho$, where $\rho$ denotes the amount adsorbed normalized to its maximum. The number of metastable states at a given $\mu$ is $\mathcal{N}(\mu ; N)=\int \mathcal{N}(\rho ; \mu ; N) d \rho$

These quantities actually strongly depend on the particular distribution of heterogeneities in the porous material. In our case, it corresponds to a given string of domains characterizing the linear pore. Any such "realization of the disorder" will be denoted by $\alpha$. As usual in disordered systems, it is interesting to consider both the average and typical number of metastable states: $\overline{\mathcal{N}_{\alpha}(\rho ; \mu ; N)}$ and $\exp \left[\overline{\ln \mathcal{N}_{\alpha}(\rho ; \mu ; N)}\right]$ where the bar denotes the average over the disorder $\alpha$. Since these quantities are both expected to scale exponentially with $N$ within the hysteresis, one introduces the corresponding so-called annealed and quenched complexities: 


$$
\begin{aligned}
& \sum_{\mathrm{A}}^{\infty}(\rho ; \mu)=\lim _{N \rightarrow \infty} \frac{1}{N} \ln \overline{\mathcal{N}_{\alpha}(\rho ; \mu ; N)} \\
& \sum_{\mathrm{Q}}^{\infty}(\rho ; \mu)=\lim _{N \rightarrow \infty} \frac{1}{N} \overline{\ln \mathcal{N}_{\alpha}(\rho ; \mu ; N)}
\end{aligned}
$$

The calculation of the finite-size annealed complexity

$$
\sum_{\mathrm{A}}^{\infty}(\rho ; \mu ; N)=\frac{1}{N} \ln \overline{\mathcal{N}_{\alpha}(\rho ; \mu ; N)}
$$

is straightforward. However, the finite-size complexity $N^{-1} \ln \mathcal{N}_{\alpha}(\rho ; \mu ; N)$ diverges each time the density of metastable states reaches zero, which may always occur for some configurations of the heterogeneities in the pore, leading to an undefined quenched complexity. To circumvent the problem, Pérez-Reche ${ }^{14}$ and collaborators proposed a method inspired by an analytical calculation, ${ }^{13}$ which consists in introducing the Legendre-Fenchel transform $\Lambda^{\alpha}(g ; \mu ; N)$ of the finite size disorder dependent complexity $N^{-1} \ln \mathcal{N}^{\alpha}(\rho ; \mu ; N)$ :

$$
\Lambda_{\alpha}(g ; \mu ; N)=\max _{\rho}\left\{\frac{1}{N} \ln \mathcal{N}_{\alpha}(\rho ; \mu ; N)+g \rho\right\}
$$

This quantity is always defined for any real value $g$, even if $\mathcal{N}^{\alpha}(\rho ; \mu ; N)$ reaches zero. Let us denote $\rho_{\alpha}(g ; \mu ; N)$ the $\rho$-value which maximizes the quantity between parentheses in Eq. 4. One has:

$$
\Lambda_{\alpha}(g ; \mu ; N)=\frac{1}{N} \ln \mathcal{N}_{\alpha}\left(\rho_{\alpha} ; \mu ; N\right)+g \rho_{\alpha}
$$

Averaging over disorder, one gets:

$$
\Lambda(g ; \mu ; N)=\frac{1}{N} \overline{\ln \mathcal{N}_{\alpha}\left(\rho_{\alpha} ; \mu ; N\right)}+g \rho(g ; \mu ; N)
$$

where $\rho(g ; \mu ; N)=\overline{\rho_{\alpha}(g ; \mu ; N)}$. The quenched complexity can thus be defined for a finite system as:

$$
\sum_{\mathrm{Q}}^{\mathrm{L}-\mathrm{F}}(\rho ; \mu ; N)=\Lambda(g(\rho ; \mu ; N) ; \mu ; N)-\rho g(\rho ; \mu ; N)
$$


where $g(\rho ; \mu ; N)$ is implicitly defined by $\rho(g ; \mu ; N) . \rho_{\alpha}(g ; \mu ; N)$ is expected to deviate from its average value $\rho$ by terms of order $1 / \sqrt{N}$. As a consequence, $\overline{\ln \mathcal{N}_{\alpha}\left(\rho_{\alpha} ; \mu ; N\right)}$ approaches $\overline{\ln \mathcal{N}_{\alpha}(\rho ; \mu ; N)}$ in the thermodynamic limit, and one has:

$$
\lim _{N \rightarrow \infty} \sum_{\mathrm{Q}}^{\mathrm{L}-\mathrm{F}}(\rho ; \mu ; N)=\sum_{\mathrm{Q}}^{\infty}(\rho ; \mu)
$$

\section{The characteristic function}

As any other quantity, the annealed and quenched complexities are expected to depend on the system size $N$. A general form was proposed: $\sum^{\infty}+a N^{-1} \ln N+b N^{-1}$ involving three free parameters which depend on $\rho$ and $\mu$. It has proven to be a fairly good estimate of the system size dependence, which can be used in a standard finite-size analysis for instance. ${ }^{14} \sum^{\infty}$ is the expected thermodynamic limit. Figure 3 shows the annealed complexity as a function of $\rho$ for $\mu=-8.55$, where the hysteresis is the largest, and for various system sizes $N=15,20,25$, and 30. Noticeable finite size effects are visible. The evolution of the maximum can be described using the above mentioned ansatz with the parameters: $\sum^{\infty}=0.25, a=0.65$, and $b=-0.64$. Similar results are obtained for the quenched complexity. Can this system size dependence be interpreted in terms of interdependence between domains? The answer is no, since a straightforward calculation shows that the annealed and quenched complexities are expected to exhibit finite size effects even if the domains are independent. A question thus arises: what contribution to system size dependence is specifically due to the interconnections between domains? To illustrate this point, we have considered pores of size $N=30$ made of the union of two independent smaller pores of size $N=15$. The calculated annealed complexity is given 
in the inset of Fig. 3. As can be seen the results are close to the fully interconnected pores with $N=30$. This suggests that the main contribution to the observed system size dependence is not due to interactions between subsystems. The aim of this section is to propose an analysis which exhibits solely the finite size effects due to domain interdependence.

The main idea underlying the average procedure over disorder is that a large system can be seen as the ensemble of many subsystems which tend to be essentially independent in the thermodynamic limit. As a consequence any additive quantity is straightforwardly averaged. This is for instance the case for the envelope of the hysteresis (main adsorption/desorption loop). The case of the distribution of metastable states within the hysteresis loop is more involved. If we denote $\mathcal{N}_{\alpha}\left(\rho ; \mu ; N_{\alpha}\right)$ the distribution of metastable states for the subsystem $\alpha$ made of $N_{\alpha}$ domains, then the distribution for the union of two independent subsystems $\alpha$ and $\beta$ is given by:

$$
\mathcal{N}_{(\alpha, \beta)}\left(\rho ; \mu ; N_{\alpha}+N_{\beta}\right)=\int \mathcal{N}_{\alpha}\left(\rho_{\alpha} ; \mu ; N_{\alpha}\right) \mathcal{N}_{\beta}\left(\rho_{\beta} ; \mu ; N_{\beta}\right) \delta\left(\rho-\frac{N_{\alpha} \rho_{\alpha}+N_{\beta} \rho_{\beta}}{N_{\alpha}+N_{\beta}}\right) d \rho_{\alpha} d \rho_{\beta}
$$

where the integral is performed over all possible values of adsorbed densities in $\alpha$ and $\beta$ subsystems, and $\delta$ is the Dirac measure. This formula can easily be generalized to more that two subsystems. Introducing the Fourier transform (characteristic function) of the distribution:

$\tilde{\mathcal{N}}_{\alpha}\left(\omega ; \mu ; N_{\alpha}\right)=\int \mathcal{N}_{\alpha}\left(\rho ; \mu ; N_{\alpha}\right) e^{i N_{\alpha} \omega \rho} d \rho$

one has:

$$
\tilde{\mathcal{N}}_{(\alpha, \beta)}\left(\omega ; \mu ; N_{\alpha}+N_{\beta}\right)=\tilde{\mathcal{N}}_{\alpha}\left(\omega ; \mu ; N_{\alpha}\right) \tilde{\mathcal{N}}_{\beta}\left(\omega ; \mu ; N_{\beta}\right)
$$

As previously, this formula can be straightforwardly generalized for more than two subsystems. This shows that the logarithm of the characteristic function is additive. We thus 
introduce $\Psi_{\alpha}\left(\omega ; \mu ; N_{\alpha}\right)=N_{\alpha}{ }^{-1} \ln \tilde{\mathcal{N}}_{\alpha}\left(\omega ; \mu ; N_{\alpha}\right)$. This quantity is expected to be selfaveraging, with fluctuations decreasing as $1 / \sqrt{N}$. The rule for averaging over disorder realizations follows immediately from the additivity relation for $\Psi_{\alpha}\left(\omega ; \mu ; N_{\alpha}\right)$ :

$$
\Psi(\omega ; \mu ; N)=\overline{\Psi_{\alpha}\left(\omega ; \mu ; N_{\alpha}\right)}
$$

where the bar denotes the average over disorder realizations $\alpha$ with identical number of domains $N_{\alpha}=N$.

Some subtleties need to be fixed regarding the numerical implementation of this average. The quantity $\tilde{\mathcal{N}}_{\alpha}\left(\omega ; \mu ; N_{\alpha}\right)$ being complex, the imaginary part of $\Psi_{\alpha}\left(\omega ; \mu ; N_{\alpha}\right)$ is defined modulo $2 \pi$. This has obviously no consequences for recovering the initial distribution for a given disorder realization (inverse Fourier transform), but might introduce spurious effects when performing the average if no care is taken to get the smooth (continuous) numerical solution for the logarithm of $\tilde{\mathcal{N}}_{\alpha}\left(\omega ; \mu ; N_{\alpha}\right)$ seen as a function of $\omega$. Note that this is almost always possible except when $\tilde{\mathcal{N}}_{\alpha}\left(\omega ; \mu ; N_{\alpha}\right)$ goes through the origin, which is quite rare and occurs only for large values of $\omega$. Figure 4 gives the Fourier transforms for two disorder realizations. The low $\omega$ part contains the main information on the shape of the distribution, while the high frequency part, which generally approaches the origin while turning around, is due to fast variations in the distributions, and is actually irrelevant (its average over disorder vanishes). The results for the average $\Psi(\omega ; \mu ; N)$ are given in Fig. 5 for various $N$ and for $\mu$ $=-8.55$. The maximum of $\Psi(\omega ; \mu ; N)$ is obtained for $\omega=0$ and corresponds to the finite-size quenched complexity associated to the total number of metastable states at $\mu=-8.55$ : $\Sigma_{\mathrm{Q}}(\mu ; N)=N^{-1} \overline{\ln \mathcal{N}(\mu ; N)}$, where $\mathcal{N}(\mu ; N)=\int \mathcal{N}(\rho ; \mu ; N) d \rho$. The imaginary part is 
essentially linear, the slope giving the density $\rho$ which maximizes the number of metastable states (maximum of complexity).

Let us now focus on the dependence of $\Psi(\omega ; \mu ; N)$ with $N$. The remarkable feature exhibited by Fig. 5 is that $\Psi(\omega ; \mu ; N)$ barely depends on the system size. By construction, $\Psi(\omega ; \mu ; N)$ does not depend on $N$ if the domains are independent. More generally, if the system can be seen as the union of independent subsystems of typical size $N_{0}$, then $\Psi(\omega ; \mu ; N)$ becomes independent of $N$ above $N_{0}$. In that sense, $\Psi(\omega ; \mu ; N)$ appears as the best quantity to perform a quantitative finite size analysis. We used the same ansatz $\sum^{\infty}+a N^{-1} \ln N+b N^{-1}$, applied to the maximum $\Psi(\omega=0 ; \mu ; N)$. A best fit is obtained for $\sum^{\infty}=0.2266, a=-0.0120$, and $b=$ 0.0735. The two parameters characterizing the $N$-dependence are two orders of magnitude smaller than for the maximum of the annealed or the quenched complexity (see previous section), leading to a much better estimate of the thermodynamic limit. The fact that the curves for $N=15$ and 30 are quite similar means that pores of length 30 can be seen as the union of independent sub-pores of size 15. This is a quantitative confirmation of the results anticipated in Fig. 3. More precisely, one notes that the curve for $N=15$ slightly differs from the other ones, but all curves for $N=20$ to 30 essentially coincide. This shows that the intrinsic system size dependence due to subsystem interconnections is quite small above $N=$ 20.

The metastable state distribution of the average system is recovered by inverse Fourier transform (the normalization is $N / 2 \pi$ ). The associated complexity

$$
\Sigma_{\mathrm{Q}}^{\mathrm{F}}(\rho ; \mu ; N)=\frac{1}{N} \ln \left\{\frac{N}{2 \pi} \int_{-\infty}^{+\infty} e^{N[\Psi(\omega ; \mu ; N)-i \rho \omega]} d \omega\right\}
$$


is given in Fig. 6 for $N=15$ to 30. The subscript Q is justified later. As can be seen, for low values of $N$ the numerical fluctuations are quite important (due to fluctuating values of $\Psi_{\alpha}(\omega ; \mu ; N)$ for $|\omega|>1$ ), but decrease for larger $N$ (as these contributions become negligible in Eq. 13).

What is the connection, in the thermodynamic limit, between $\Sigma_{\mathrm{Q}}^{\mathrm{F}}(\rho ; \mu ; N)$ and the previously introduced complexities? Figure 7 shows a superimposition of the various complexities for $N$ $=30$. As can be seen, $\sum_{\mathrm{Q}}^{\mathrm{F}}(\rho ; \mu ; N)$ almost coincide with $\sum_{\mathrm{Q}}^{\mathrm{L}-\mathrm{F}}(\rho ; \mu ; N)$ and one may thus expect that $\Sigma_{\mathrm{Q}}^{\mathrm{F}}(\rho ; \mu ; N)$ converges to the quenched complexity in the thermodynamic limit. More formally, the link between $\Sigma_{\mathrm{Q}}^{\mathrm{F}}(\rho ; \mu ; N)$ and the usual quenched complexity can be rationalized as follows. We introduce the generalized Laplace Transform $\mathcal{L} \mathcal{N}_{\alpha}$ of $\mathcal{N}_{\alpha}$ as:

$$
\mathcal{L N}_{\alpha}\left(z ; \mu ; N_{\alpha}\right)=\int \mathcal{N}_{\alpha}\left(\rho ; \mu ; N_{\alpha}\right) e^{N_{\alpha} \rho z} d \rho
$$

with $z$ any complex number (the integral is always defined). Following a route similar to the previously introduced Fourier transform, it is straightforward to show that the natural quantity to be averaged over disorder is $\Phi_{\alpha}\left(z ; \mu ; N_{\alpha}\right)=N_{\alpha}^{-1} \ln \mathcal{L} \mathcal{N}_{\alpha}\left(z ; \mu ; N_{\alpha}\right)$, and we define $\Phi(z ; \mu ; N)=\overline{\Phi_{\alpha}\left(z ; \mu ; N_{\alpha}\right)}$, where the average is performed as previously on system realizations with $N$ domains. Note that $\Psi(\omega ; \mu ; N)=\Phi(i \omega ; \mu ; N)$. For real values of $z=g$ the leading term (for $N_{\alpha}$ large) of $\Phi_{\alpha}\left(g ; \mu ; N_{\alpha}\right)$ can be easily calculated using the Laplace method (the integral of the exponentially large term is dominated by its largest value):

$$
\Phi_{\alpha}\left(g ; \mu ; N_{\alpha}\right)=\max _{\rho}\left\{\frac{1}{N_{\alpha}} \ln \mathcal{N}_{\alpha}\left(\rho ; \mu ; N_{\alpha}\right)+g \rho\right\}+\mathrm{o}(1)
$$

One recognizes in the right hand side the Legendre transform $\Lambda_{\alpha}\left(g ; \mu ; N_{\alpha}\right)$ of $N_{\alpha}^{-1} \ln \mathcal{N}_{\alpha}\left(\rho ; \mu ; N_{\alpha}\right)$ (Eq. 4), which allowed to define $\sum_{\mathrm{Q}}^{\mathrm{L}-\mathrm{F}}(\rho ; \mu ; N)$. The logarithm of the 
Laplace transform being self-averaging, one expects the Legendre transform and the quenched complexity to be self-averaging too. In the thermodynamic limit, $\Psi(\omega ; \mu ; N)$ and $\Lambda(g ; \mu ; N)$ are the restrictions of the same analytic function $\Phi(z ; \mu ; N)$ on the imaginary and real axes respectively. The associated complexities, defined as inverse transformations, are thus expected to be identical in the thermodynamic limit. Hence $\lim _{N \rightarrow \infty} \sum_{\mathrm{Q}}^{\mathrm{F}}(\rho ; \mu ; N)=\sum_{\mathrm{Q}}^{\infty}(\rho ; \mu)$.

The two approaches however differ for finite system size. The Fourier transform (or equivalently the Laplace transform) is exact, whereas the Legendre transform appears as an approximation for large $N$. The second method is easier to handle, and closely follows the definition of the quenched complexity in finite systems. It however introduces important contributions of order $N^{-1} \ln N$ and $N^{-1}$ making the finite size analysis less informative. Note that in principle these contributions could be calculated. On the other hand, the Fourier transform is more involved and more sensitive to numerical inaccuracies (especially when inversion back to the density of metastable states is concerned), but is perfectly suited to study finite size dependence.

\section{Extrapolation to the thermodynamic limit}

Since the intrinsic system size dependence due to subsystem interconnections has proven to be quite small, it is possible to propose a good approximation for the thermodynamic limit. Let us consider a large system made of $n$ independent subsystems of size $N$ ( $n$ is for instance the number of disorder realizations used in the previous averages, but we actually can take it as large as we want to reach the thermodynamic limit). The total number of domains is $n N$. 
Following the additivity rule for $\Psi_{\alpha}(\omega ; \mu ; N)$, the complexity associated to the distribution of metastable states in the thermodynamic limit is given by:

$$
\sum_{\mathrm{Q}}^{\mathrm{F}, \infty}(\rho ; \mu ; N)=\lim _{n \rightarrow \infty} \frac{1}{n N} \ln \left\{\frac{n N}{2 \pi} \int_{-\infty}^{+\infty} e^{n N[\Psi(\omega ; \mu ; N)-i \rho \omega]} d \omega\right\}
$$

Note that this definition differs from Eq. 13: $\sum_{\mathrm{Q}}^{\mathrm{F}, \infty}(\rho ; \mu ; N)$ is the thermodynamic limit complexity of a system made of the union of independent subsystems of size $N$. Since $\Psi(\omega ; \mu ; N)$ is almost independent of $N, \sum_{\mathrm{Q}}^{\mathrm{F}, \infty}(\rho ; \mu ; N)$ is expected to be very close to the true thermodynamic limit $\sum_{\mathrm{Q}}^{\infty}(\rho ; \mu)$. Let us examine Eq. 16 for the particular value of $\rho$ corresponding to the slope of the argument of $\Psi(\omega ; \mu ; N)$. This choice is expected to give the maximum of the complexity. For this choice $\Sigma_{\mathrm{Q}}^{\mathrm{F}, \infty}(\rho ; \mu ; N)$ is equal to the maximum of $\Psi(\omega ; \mu ; N)$, which is obtained for $\omega=0$ and equals the quenched complexity $\Sigma_{\mathrm{Q}}(\mu ; N)$, which is precisely the expected limit for the maximum of the complexity. For other values of $\rho$ the calculations are more involved and cannot be performed numerically straightforwardly.

An analytical approach is expected to be much more efficient and precise. This can be performed for instance for the simple binomial curves obtained for the model with independent domains (ID). These curves can then be used as test functions to fit the data, and deduce the thermodynamic limit. The binomial curves are defined as follows: For $N$ independent domains at a given chemical potential $\mu$, if we suppose that each domain can be in one of two states (empty or filled), and that each filled domain contributes $1 / N$ of the total adsorbed density (equal to 1 at saturation), then the corresponding distribution $\mathcal{N}^{\mathrm{ID}}(\rho ; \mu ; N)$ is simply given by the binomial coefficients as:

$$
\mathcal{N}^{\mathrm{ID}}(\rho ; \mu ; N)=\sum_{k=0}^{N} \frac{N !}{k !(N-k) !} \delta\left(\rho-\frac{k}{N}\right)
$$


where $\delta$ is Dirac measure. Note that it is possible to smear out the delta functions over $\rho$-bins, of width $1 / N$ for instance, which is simply equivalent to take into account a dispersion in the amount adsorbed per domain at saturation. In this ID model, the total number of metastable states at the given $\mu$ is $\mathcal{N}^{\mathrm{ID}}(\mu ; N)=2^{N}$. A more realistic model should however take into account the fact that some of the domains may actually be in a single state (due to the particular value of the fluid/wall interactions in these domains), and that for the domains which can be in two states (bi-stable domains), the gaslike state is generally not completely empty, and the liquidlike state has a density generally less than 1 . We are thus lead to consider the phenomenological distribution

$$
\mathcal{N}^{\text {phen. }}(\rho ; \mu ; N)=\frac{1}{\left(\rho^{+}-\rho^{-}\right)} \mathcal{N}^{\mathrm{ID}}\left(\frac{\rho-\rho^{-}}{\rho^{+}-\rho^{-}} ; \mu ; \xi N\right)
$$

where $\xi$ is the proportion of bi-stable domains and $\left[\rho^{-}, \rho^{+}\right]$is the interval where the density of metastable states is not zero. The normalization follows from the Dirac measure. This phenomenological distribution is thus defined by 3 independent parameters: $\rho^{-}, \rho^{+}$and $\xi$. After calculating the Fourier transform, one gets:

$$
\Psi^{\text {phen. }}(\omega ; \mu ; N)=\xi \ln \left\{2 \cos \left(\omega \frac{\rho^{+}-\rho^{-}}{2 \xi}\right)\right\}+i \omega \frac{\rho^{+}+\rho^{-}}{2}
$$

The three free parameters are determined by least squares method and given in Table 1 . The corresponding curves for $N=30$ are given in Fig. 5 and 6 (dotted lines). Note that in Fig. 6, for clarity, the discrete peaks associated to the delta functions in Eq. 17-18 have been smeared out over the bins in density. As can be seen in Fig. 5, a fairly good agreement is obtained around the maximum of $\Psi(\omega ; \mu ; N)$, while the large $\omega$ behavior is not correctly reproduced. These large $\omega$ contributions are actually associated to sharp variations of the density of metastable states and neglecting them is equivalent to smoothing the data. It can be seen on 
Fig. 6 that these contributions are not relevant since the agreement for the density of metastable states is very good. The advantage of this treatment is that one can now give the analytic expression for the thermodynamic limit (using Eqs 16, 18 and Stirling formula):

$$
\sum_{\mathrm{Q}}^{\mathrm{F}, \infty}(\rho ; \mu ; N)=-\xi\left\{\frac{\rho-\rho^{-}}{\rho^{+}-\rho^{-}} \ln \frac{\rho-\rho^{-}}{\rho^{+}-\rho^{-}}+\frac{\rho^{+}-\rho}{\rho^{+}-\rho^{-}} \ln \frac{\rho^{+}-\rho}{\rho^{+}-\rho^{-}}\right\}
$$

where the $N$ dependence originates from the 3 parameters. The results for the various system sizes are shown in Fig. 8. The remarkable feature is that the residual finite size effect is now quite small compared to the one observed for the Legendre-Fenchel quenched complexity (Eq. 7, shown as dotted lines for comparison) and properly measures the effect of interconnections between domains. In particular for $\rho<0.6$ the thermodynamic limit behavior seems to be reached even for $N=15$, in close agreement with previous conclusion. The maximum is also quite stable, very close to its theoretical thermodynamic limit 0.22557 (the difference is 1.5 percent) ${ }^{20}$ Beside these successes, one however notes failures around $\sum_{\mathrm{Q}}^{\mathrm{F}, \infty}(\rho ; \mu ; N)=0$. Both complexities $\sum_{\mathrm{Q}}^{\mathrm{L}-\mathrm{F}}(\rho ; \mu ; N)$ and $\sum_{\mathrm{Q}}^{\mathrm{F}, \infty}(\rho ; \mu ; N)$ do not seem to reach zero for the same limiting $\rho$-values. This is clearly visible for the low $\rho$ bounds since they are essentially independent of system size, but remains true for the high $\rho$ bound. In these regions the complexities vary rapidly, and one does not expect $\sum_{\mathrm{Q}}^{\mathrm{F}, \infty}(\rho ; \mu ; N)$ to be very accurate because the quality of the fit is questionable for large $\omega$ values (see Fig. 5).

The same analysis and fitting procedure has been performed for all chemical potential values. The result is given in Fig. 9. The main averaged adsorption/desorption isotherm is also given. As can be seen, $\Sigma_{\mathrm{Q}}^{\mathrm{F}, \infty}(\rho ; \mu ; N)$ is positive within the hysteresis and reaches its maximum at the center of the adsorption/desorption loop, where the density of metastable states is maximal. As previously shown for $\mu=-8.55$, the complexity, as given in Fig. 9, is 
quantitative within few percent around the line of maxima. The uncertainty grows larger when approaching the region where $\sum_{\mathrm{Q}}^{\mathrm{F}, \infty}(\rho ; \mu ; N)=0$. This can be seen on Fig. 9 since $\sum_{\mathrm{Q}}^{\mathrm{F}, \infty}(\rho ; \mu ; N)$ does not reach zero exactly on the main adsorption/desorption isotherm. ${ }^{13}$ It is also possible to impose that $\rho^{-}$and $\rho^{+}$be explicitly the expected main average adsorption and desorption densities. In this case, one gets a quantitative determination of the quenched complexity in the vicinity of the hysteresis loop but also around its maximum. We however expect to improve the numerical treatment accuracy in order to be able to show that $\sum_{\mathrm{Q}}^{\mathrm{F}, \infty}(\rho ; \mu ; N)$ vanishes exactly on the main adsorption loop. This could be achieved using better trial functions able to fit the characteristic function data on a larger range.

\section{Conclusion}

The disorder-induced adsorption/desorption hysteresis has been studied in the particular case of a simple Lennard-Jones fluid confined in a linear inhomogeneous pore. The randomly distributed physico-chemical heterogeneities, associated to domains, induce a complex free energy landscape, and consequently metastable states. Our approach proceeds in two steps: we first calculate "conditional isotherms" by molecular simulation, which describe the fluid state in a given domain as a function of the chemical potential and for the various possible fluid states in the neighboring domains. Once these data are known, algorithms are used to calculate the adsorption/desorption isotherms for the entire pore, or enumerate all possible metastable states. Their number is shown to grow exponentially with system size (number of heterogeneities), and one thus introduces the complexities (logarithm normalized to system size). The difficult issue regarding the average over disorder is discussed. One generally 
introduces the annealed and quenched complexities, but we show that the characteristic function, associated to the distribution of metastable states, can straightforwardly be averaged over disorder. The associated complexity yields the quenched complexity in the thermodynamic limit.

As usual in numerical approaches, a finite size analysis is required to address the thermodynamic limit. The annealed and quenched complexities exhibit substantial finite size effects, most of which being irrelevant regarding the interconnections between domains: most of these effects would arise even if the domains were independent. To disentangle these contributions, the characteristic function associated to the distribution of metastable states is shown to be the best approach. Its main property is that, by construction, it is expected to be independent of system size for $N>N_{0}$, if subsystems of size $N_{0}$ are independent. Its dependence with $N$ thus solely measures the effect of the interconnections between domains of size $N$. The calculations for our linear pore model clearly show that the annealed and quenched complexities exhibit finite size effect, while the characteristic function is essentially independent of system size above 20 domains. This shows that the interconnections can be neglected between pore sub-regions containing more than 20 domains. This important information can be exploited to determine the thermodynamic limit, since it is possible to calculate exactly the distribution of metastable states for a system made of an arbitrarily large number of independent sub-regions of size 20, 25 or 30 domains (for which exact enumeration was previously performed). The maximum of the distribution, which can straightforwardly be obtained, is very close to the expected theoretical limit (within $1.5 \%$ ). The other cases however require to perform an inverse Fourier transform with an arbitrarily large exponent, which introduces numerical noise. We thus introduced a collection of $\mathrm{N}$ dependent, analytically tractable test-functions used to perform best fits with the simulation 
data. The obtained quenched complexity (in the thermodynamic limit) takes maximal values in the center of the hysteresis loop, in quantitative agreement (within few percent) with previous theoretical calculations performed for some particular points. The uncertainties increase in the boarder region where the complexity reaches zero. The results are however consistent with the usual picture that the quenched complexity reaches zero on the main adsorption/desorption hysteresis loop. Noticeable improvement is expected with better trial functions able to fit the characteristic function data on a larger range. This approach offers a helpful framework to perform quantitative finite size study and extrapolation to the thermodynamic limit, relevant for many hysteresis phenomena, including adsorption and magnetic materials.

Acknowledgement: Dr. E. Kierlik and Dr. G. Tarjus are gratefully acknowledged for helpful discussions. The simulations were performed thanks to grant from the Institut de Développement des Ressources en Informatique Scientifique IDRIS-CNRS, Orsay, France. 


\section{References}

1 B. Coasne, A. Grosman, C. Ortega, and M. Simon, Phys. Rev. Lett. 88 (25), 256102 (2002).

2 L. Bruschi, G. Fois, G. Mistura, K. Sklarek, R. Hillebrand, M. Steinhart, and U. Gösele, Langmuir 24 (19), 10936 (2008).

3 L. Bruschi and G. Mistura, J. Low Temp. Phys. 157, 206 (2009).

4 D. Wallacher, N. Künzner, D. Kovalev, N. Knorr, and K. Knorr, Phys. Rev. Lett. 92 (19), 195704 (2004).

5 E. Kierlik, P. A. Monson, M. L. Rosinberg, L. Sarkisov, and G. Tarjus, Phys. Rev. Lett. 87 (5), 055701 (2001).

6 E. Kierlik, M. L. Rosinberg, G. Tarjus, and P. Viot, Phys. Chem. Chem. Phys. 3 (7), $1201(2001)$.

7 E. Kierlik, P. A. Monson, M. L. Rosinberg, and G. Tarjus, J. Phys.: Condens. Matter 14, 9295 (2002).

J. Puibasset, J. Phys. Chem. B 109 (10), 4700 (2005).

J. Puibasset, J. Chem. Phys. 127 (15), 154701 (2007).

J. Puibasset, Langmuir 25 (2), 903 (2009).

S. Naumov, A. Khokhlov, R. Valiullin, J. Kärger, and P. A. Monson, Phys. Rev. E 78 (6), 060601 (2008).

E. Kierlik, J. Puibasset, and G. Tarjus, J. Phys.: Condens. Matter 21 (15), 155102 (2009).

F. Detcheverry, M. L. Rosinberg, and G. Tarjus, Eur. Phys. J. B 44, 327 (2005).

F. J. Pérez-Reche, M. L. Rosinberg, and G. Tarjus, Phys. Rev. B 77 (6), 064422 (2008).

M. L. Rosinberg, G. Tarjus, and F. J. Pérez-Reche, J. Stat. Mech. (03), P03003 (2009). 
A. Grosman and C. Ortega, Langmuir 24 (8), 3977 (2008).

A. Grosman and C. Ortega, Phys. Rev. B 78, 085433 (2008).

A. Grosman and C. Ortega, Langmuir 25 (14), 8083 (2009).

L. Bruschi, G. Mistura, L. Liu, W. Lee, U. Gösele, and B. Coasne, Langmuir 26 (14), 11894 (2010).

J. Puibasset, J. Chem. Phys. 133 (10), 104701 (2010). 


\section{Tables}

Table 1: Values for $\xi, \rho^{-}$, and $\rho^{+}$for various $N$ used to fit $\Psi(\omega ; \mu ; N)$ (see Fig. 5) with Eq. 19.

\begin{tabular}{cccc}
\hline \hline$N$ & $\xi$ & $\rho^{-}$ & $\rho^{+}$ \\
\hline 15 & 0.3315 & 0.2235 & 0.8666 \\
20 & 0.3299 & 0.2244 & 0.8916 \\
25 & 0.3293 & 0.2275 & 0.9045 \\
30 & 0.3287 & 0.2284 & 0.9156 \\
\hline \hline
\end{tabular}




\section{Figure captions}

Figure 1: Left: schematic representation of a pore exhibiting disorder. The pore is made of $N$ domains with variable affinity for the adsorbed fluid. For each domain, the width represents this affinity, obtained as a modulation of the intensity of the fluid/wall interaction with respect to its standard value given by the argon / oxygen energetic Lennard-Jones parameter (scale given in the right panel).

Figure 2: Typical example of adsorption/desorption hysteresis for a simple fluid adsorbed in an inhomogeneous linear pore presenting 20 domains (variation of the fluid/wall interaction between domains as a source of disorder). Continuous line (black): main adsorption/desorption hysteresis loop. Short lines (red): all metastable states. The 405 metastable states are distributed over the whole hysteresis, with highest density in the center.

Figure 3: Symbols: annealed complexity (Eq. 1) versus amount adsorbed for various system sizes (given in the figure) and $\mu=-8.55$ (lines are guides to the eye). Insert: Circles: $N=15$; triangles: $N=30$; crosses: pores of size $N=30$ made of the union of two independent pores of size $N=15$. The main panel exhibits the finite size effect, while the insert helps to evaluate the hypothesis of independence between subsystems.

Figure 4: Upper panel: Fourier transform $\tilde{\mathcal{N}}_{\alpha}\left(\omega ; \mu ; N_{\alpha}\right)$ (Eq. 10) in the complex plane for two particular disorder realizations and system sizes (black: $N=15$, red: $N=30$ ). Lower panels: real and imaginary parts of $\Psi_{\alpha}\left(\omega ; \mu ; N_{\alpha}\right)=N_{\alpha}^{-1} \ln \tilde{\mathcal{N}}_{\alpha}\left(\omega ; \mu ; N_{\alpha}\right)$ for the same disorder realizations. 
Figure 5: Lines: real (upper panel) and imaginary (lower panel) parts of $\Psi(\omega ; \mu ; N)$ (Eq. 12) as a function of $\omega$ for various system sizes and for $\mu=-8.55$. Dotted line: best fit for $N=30$ with Eq. 19.

Figure 6: $\sum_{\mathrm{Q}}^{\mathrm{F}}(\rho ; \mu ; N)$ (Eq. 13) versus $\rho$ for $N=15,20,25,30$ and $\mu=-8.55$. Dotted line: complexity derived from Eq. 18 with the best-fit parameters given in Table 1 for $N=30$. The delta peaks are smeared over the density bins.

Figure 7: Superimposition of the various complexities (Eqs. 3, 7 and 13) versus $\rho$ for $N=30$ and $\mu=-8.55$

Figure 8: Solid lines: $\sum_{\mathrm{Q}}^{\mathrm{F}, \infty}(\rho ; \mu ; N)$ for $N=15,20,25$, and 30 and $\mu=-8.55$ (Eq. 20 and Table 1). Dotted lines: $\sum_{\mathrm{Q}}^{\mathrm{L}-\mathrm{F}}(\rho ; \mu ; N)$ (Eq. 7) for the same system sizes.

Figure 9: $\sum_{\mathrm{Q}}^{\mathrm{F}, \infty}(\rho ; \mu ; N)$ for $N=30$ (Eqs. 18, 19, and 20). Upper panel: 3D representation. Lower panel: in-plane representation; the solid line is the main averaged adsorption/desorption isotherm. 